\title{
Absence of platelet-activating factor receptor protects mice from osteoporosis following ovariectomy
}

\author{
Hisako Hikiji, ${ }^{1,2}$ Satoshi Ishii, ${ }^{2}$ Hideo Shindou, ${ }^{2}$ Tsuyoshi Takato,, ${ }^{1}$ and Takao Shimizu² \\ 1Department of Oral and Maxillofacial Surgery and 2Department of Biochemistry and Molecular Biology, Faculty of Medicine, \\ The University of Tokyo, Bunkyo, Tokyo, Japan.
}

\begin{abstract}
While platelet-activating factor (PAF) is produced in various diseases associated with bone resorption, its functions in bone metabolism remain unknown. Using PAF receptor-deficient mice, we evaluated the role of PAF in the development of bone resorption following ovariectomy, a model of postmenopausal osteoporosis. Through observations of bone mineral density and histomorphometric parameters, it was found that bone resorption was markedly attenuated in PAF receptor-deficient mice, indicating that PAF links estrogen depletion and osteoporosis in vivo. Osteoclasts expressed higher amounts of the enzymes required for PAF biosynthesis than osteoblasts. TNF- $\alpha$ and IL-1 $\beta$ increased the acetyl-coenzyme A:lyso-PAF acetyltransferase activity in osteoclasts. Osteoclasts, but not osteoblasts, expressed the functional PAF receptor. PAF receptor stimulation prolonged the survival of osteoclasts in vitro. Furthermore, osteoclasts treated with a PAF receptor antagonist, and also those from PAF receptor-deficient mice, showed reductions in survival rate and Ca resorption activity. Consistently, in organ cultures, bone resorption was significantly suppressed by a PAF receptor antagonist treatment or genetic PAF receptor deficiency. Thus, these results suggest that, through the inflammatory cytokines, estrogen depletion enhances PAF production as a unique autocrine factor for osteoclast functions. Inhibition of PAF function might pave the way for a new strategy to prevent postmenopausal bone loss without disturbing osteoblast functions.
\end{abstract}

\section{Introduction}

Postmenopausal osteoporosis is a condition caused principally by an acute decrease in serum estrogen levels after cessation of ovarian function. Estrogen deficiency results in an increase in bone turnover (1) and a bone-remodeling imbalance, leading to bone resorption and an increased risk of fracture. Approximately 100 million women worldwide suffer from postmenopausal osteoporosis, and therapies such as hormone replacement therapy have been widely tested as prevention methods (2-4).

Platelet-activating factor (PAF, 1-O-alkyl-2-acetyl-sn-glycero-3phosphocholine) is a potent phospholipid mediator with various biologic activities, including platelet activation, airway constriction, hypotension, and vascular permeation $(5,6)$ via activation of a G-protein-coupled PAF receptor (PAFR) $(7,8)$. It has been reported that PAF is produced in inflamed human gingival tissue (9) and detected in the arthritic joint fluids of rabbits with acute antigeninduced arthritis (10). Peripheral plasma concentrations of PAF were also noted to be high in patients suffering from hypercalcemia of malignancy (11). Thus, PAF may be implicated in diseases asso-

Nonstandard abbreviations used: acetyl-CoA:lyso-PAF acetyltransferase (lyso-PAF acetyltransferase); acetyl-coenzyme A (acetyl-CoA); 4-amidinophenylmethanesulfonyl fluoride (APMSF); bone mineral density (BMD); cytosolic phospholipase $\mathrm{A}_{2} \alpha$ (cPLA $2 \alpha) ; 1,25$-dihydroxyvitamin D3 $\left(1,25-[\mathrm{OH}]_{2} \mathrm{D}_{3}\right)$; dual x-ray absorptiometry (DXA); 1-O-hexadecyl-sn-glycero-3-phosphocholine (lyso-PAF); intracellular $\mathrm{Ca}^{2+}$ concentration $\left(\left[\mathrm{Ca}^{2+}\right]_{i}\right)$; methylcarbamoyl PAF (mc-PAF); osteoclast surface per bone surface (Oc.S/BS); osteoid thickness (O.Th); platelet-activating factor (PAF); PAF receptor (PAFR); PAFR knockout (PAFR-KO); PAFR wild type (PAFR-WT); receptor activator of NF-KB ligand (RANKL); tartrate-resistant acid phosphatase (TRAP); trabecular bone volume (BV/TV); trabecular number (Tb.N); trabecular separation (Tb.Sp).

Conflict of interest: The authors have declared that no conflict of interest exists.

Citation for this article: J. Clin. Invest. 114:85-93 (2004).

doi:10.1172/JCI200420504. ciated with bone resorption. Rabbit osteoclasts responded to PAF in vitro with an increase in bone pit area as well as an elevation in intracellular $\mathrm{Ca}^{2+}$ concentration $\left(\left[\mathrm{Ca}^{2+}\right]_{i}\right)$ and a transient retraction of pseudopods (12). In spite of this circumstantial evidence of PAF actions on bone metabolism, the precise role of PAF in postmenopausal osteoporosis remains unclear. By using PAFR knockout (PAFR-KO) mice, we show here the important role of PAF in the development of osteoporosis in vivo. Several in vitro data also illustrate the role of PAF as an autocrine activator of osteoclasts.

\section{Results}

Bone density in ovariectomized PAFR-KO mice. In soft $\mathrm{x}$-ray radiograph analysis, radiolucency of the excised femurs and tibiae was increased in ovariectomized PAFR-WT mice compared with sham-operated PAFR-WT mice (Figure 1A). However, ovariectomized PAFR-KO mice showed little increase in radiolucency. To quantitate these observations, the bone mineral density (BMD) of the entire femurs in the ovariectomized mice was compared with that of the sham-operated mice. Differences in BMD observed by dual x-ray absorptiometry (DXA) measurements were significant between the ovariectomized group and sham-operated group in PAFR-WT mice (Figure 1B). DXA analysis also demonstrated that the BMD of the femurs in ovariectomized PAFR-KO mice was comparable to that in sham-operated PAFR-KO mice. There were no differences in the levels of $\mathrm{Ca}$, phosphate, or parathyroid hormone in the sera of both genotypes, either with or without ovariectomy (data not shown).

Bone histomorphometry in ovariectomized PAFR-KO mice. It was apparent from the histologic observations that the bone volume in the metaphyseal region of the tibiae was reduced in ovariectomized PAFR-WT mice, but ameliorated in ovariectomized PAFR- 
A

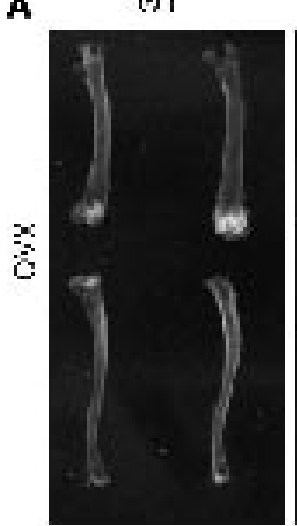

ko

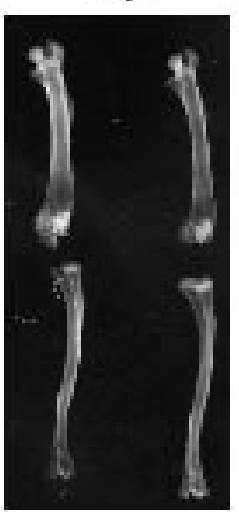

w

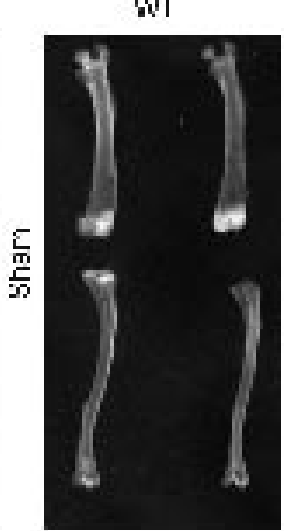

$\langle i$

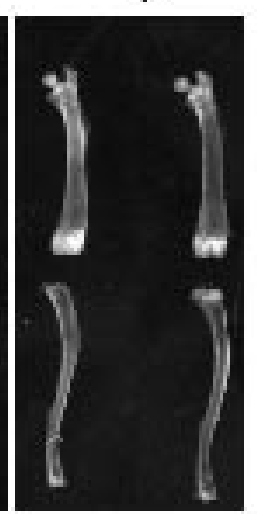

B

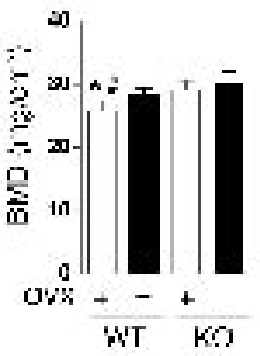

\section{Figure 1}

Radiographic bone analysis. (A) Radiographic images of femoral and tibial bones taken 4 weeks after ovariectomy. PAFR-KO and PAFRWT mice were sham-operated (Sham) or ovariectomized (OVX). Following ovariectomy, radiolucency of the excised femurs and tibiae was increased in PAFR-WT mice but not in PAFR-KO mice. (B) Measurements of BMD. The BMD of the femurs of PAFR-KO and PAFR-WT mice were measured with DXA 4 weeks after surgery. The ovariectomy-induced reduction of BMD was prevented in PAFR-KO mice. WT-OVX: $n=6$ animals; WT-sham: $n=7$ animals; KO-OVX: $n=5$ animals; KO-sham: $n=6$ animals. Values are given as mean \pm SD. ${ }^{*} P<0.005$ vs. KO-OVX; ${ }^{P}<0.05$ vs. WT-sham.

KO mice, compared with sham-operated mice (Figure 2A). Histomorphometric analysis of the metaphyseal region in the tibial bone confirmed these results (Figure $2 \mathrm{~B}$ ). Trabecular bone volume $(\mathrm{BV} / \mathrm{TV})$ was significantly reduced in PAFR-WT mice, but not in PAFR-KO mice, by ovariectomy. Other indices related to BV/TV, trabecular number (Tb.N), and trabecular separation (Tb.Sp) also indicated that the bone volume of ovariectomized PAFR-KO mice was amended to the level of the sham-operated PAFR-KO mice. The reduction in bone mass in PAFR-WT mice was associated with an increase in bone turnover because osteoid thickness (O.Th) and osteoclast surface per bone surface (Oc.S/BS) mice were increased significantly in ovariectomized PAFR-WT relative to sham-operated PAFR-WT mice.

Acetyl-CoA:lyso-PAF acetyltransferase activity and cytosolic phospholipase $A_{2} \alpha$ expression in bone cells. To understand the cell lineages that have the potential for PAF synthesis in bone tissues, the activity of acetyl-CoA:lyso-PAF acetyltransferase (lyso-PAF acetyltransferase), which catalyzes the final reaction for PAF synthesis in the remodeling pathway (13), was measured in cultured bone cells (Figure 3A). Osteoclasts derived from RAW 264.7 mouse macrophage cells,
A
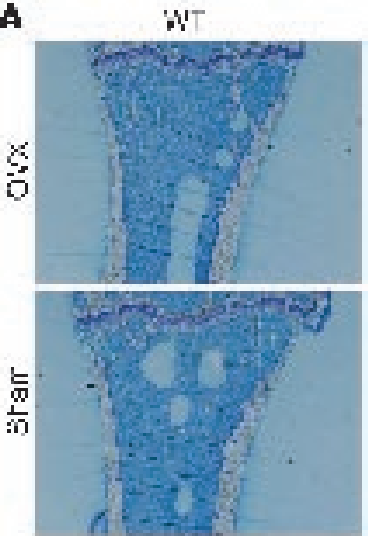

KO

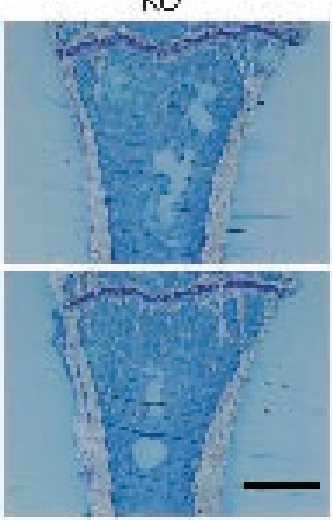

B

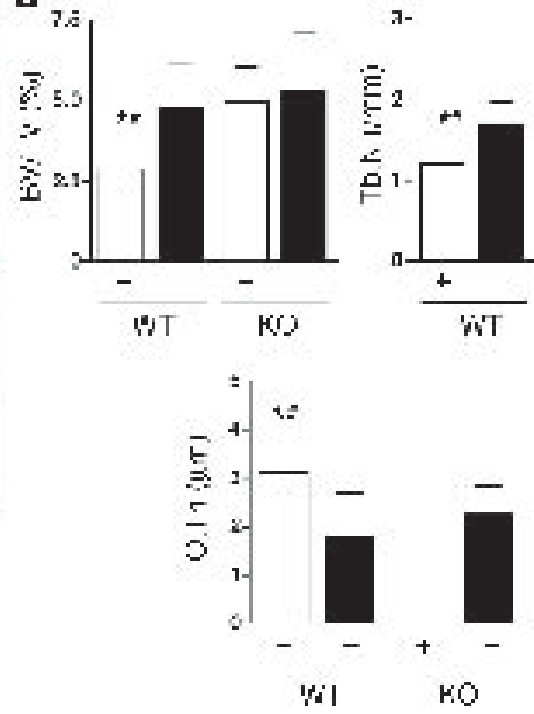

1520 -

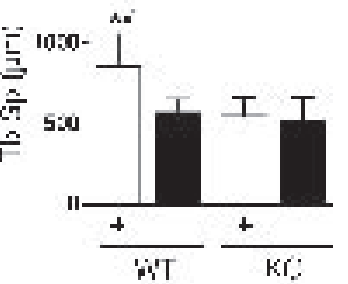

75-

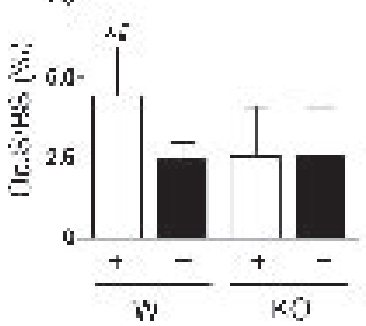

Figure 2

Prevented bone loss and unaltered bone turnover in ovariectomized PAFR-KO mice. (A) Representative toluidine blue sections of the metaphyseal region in the excised tibiae. Scale bar: $1.0 \mathrm{~mm}$. (B) Bone histomorphometry. The ovariectomy-induced reductions in BV/TV and Tb.N were prevented in PAFR-KO mice. The ovariectomy-induced increase in Tb.Sp was also prevented in PAFR-KO mice. O.Th and Oc.S/BS in WT-OVX mice were significantly increased compared with values seen in KO-OVX mice, suggesting that the reduction in bone mass in PAFR-WT mice was associated with an increase in bone turnover. Values are mean \pm SD. WT-OVX: $n=6$ animals; WT-sham: $n=7$ animals; KO-OVX: $n=5$ animals; KO-sham: $n=6$ animals. ${ }^{*} P<0.05$ vs. KO-OVX; $P<0.05$ vs. WT-sham. 


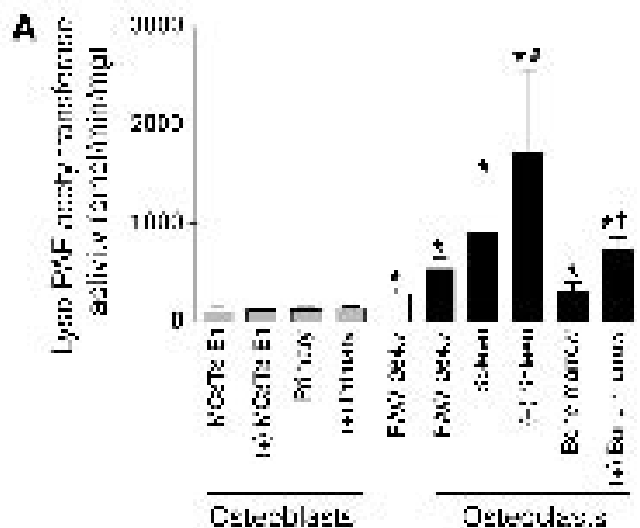

B cPI AN-

spleen-derived osteoclasts, and bone marrow-derived osteoclasts had high lyso-PAF acetyltransferase activities. Treatment with TNF- $\alpha$ and IL-1 $\beta$ increased enzyme activities significantly in both spleenand bone marrow-derived osteoclasts. On the other hand, MC3T3E1 mouse osteogenic cells and primary mouse osteoblasts displayed significantly lower activities than osteoclasts, even after cytokine stimulation. Western blot analysis showed that primary osteoclasts expressed higher amounts of cytosolic phospholipase $\mathrm{A}_{2} \alpha\left(\mathrm{CPLA}_{2} \alpha\right)$ than the osteoblasts (Figure $3 \mathrm{~B}$ ); $\mathrm{CPLA}_{2} \alpha$ is an important $\mathrm{PLA}_{2}$ for lyso-PAF production in the remodeling pathway $(14,15)$.

Expression of PAF receptor in bone cells. To analyze cell lineages expressing PAFR mRNA in bone tissues, Northern blot analysis was performed in cultured bone cells (Figure 4). Osteoclasts derived from RAW 264.7 cells, spleen-derived osteoclasts, and bone marrow-derived osteoclasts accumulated significant amounts of PAFR mRNA. Consistently, PAF raised the $\left[\mathrm{Ca}^{2+}\right]_{i}$ level in spleenderived osteoclasts of PAFR-WT mice (Figure 5A) but not in those of PAFR-KO mice (Figure 5B). In contrast, MC3T3-E1 osteoblastic cells and primary mouse osteoblasts expressed much smaller amounts of PAFR mRNA (Figure 4). Even after cytokine stimulation, PAFR mRNA expression was not induced in MC3T3-E1 cells. Furthermore, in the primary osteoblasts, PAFR activation neither elevated $\left[\mathrm{Ca}^{2+}\right]_{i}$ (Figure $5 \mathrm{C}$ ) nor induced receptor activator of NF-кB ligand (RANKL) mRNA expression (Figure 5D).

Effects of PAFR on osteoclast survival and Ca resorption. Purified mature osteoclasts readily underwent apoptosis $(16,17)$. Thus, the effect

\section{Figure 4}

Northern blot analysis of PAFR mRNA in bone cells. MC3T3-E1 cells and primary mouse osteoblasts expressed little PAFR mRNA. Even after stimulation with TNF- $\alpha$ and IL-1 $\beta$, MC3T3-E1 cells did not increase mRNA expression as denoted by $(+)$. On the contrary, RAW 264.7 cells, osteoclasts derived from RAW 264.7 cells, spleen-derived osteoclasts, and bone marrow-derived osteoclasts had high amounts of PAFR mRNA. Total RNA (10 $\mu \mathrm{g})$ was applied in each lane. Similar results were obtained in another experiment.

\section{Figure 3}

Lyso-PAF acetyltransferase activity and $\mathrm{CPLA}_{2} \alpha$ expression in bone cells. (A) Lyso-PAF acetyltransferase activity. MC3T3-E1 mouse osteogenic cells and primary mouse osteoblasts (gray bars) had low lyso-PAF acetyltransferase activities even after stimulation with TNF- $\alpha$ and IL- $1 \beta$ as designated by $(+)$. Osteoclasts derived from RAW 264.7 cells, spleen-derived osteoclasts, and bone marrowderived osteoclasts (black bars) had significantly higher lyso-PAF acetyltransferase activities compared with osteoblasts. In addition, cytokine stimulation, denoted by $(+)$, raised the activities. Values are mean \pm SD measured in triplicate or quadruplicate. Similar results were obtained in another experiment. ${ }^{*} P<0.05$ vs. primary osteoblasts; ${ }^{\#} P<0.05$ vs. unstimulated spleen-derived osteoclasts; ${ }^{\dagger} P<0.05$ vs. unstimulated bone marrow-derived osteoclasts. (B) Western blot analysis of $\mathrm{cPLA}_{2} \alpha$. The cell lysates (16 $\mu \mathrm{g}$ of protein) were subjected to SDS-PAGE and immunoblotted with an anti-mouse $C P L A_{2} \alpha$ monoclonal antibody. Spleen-derived osteoclasts and bone marrowderived osteoclasts expressed higher amounts of $c P L A_{2} \alpha$ than primary osteoblasts. Similar results were obtained in another experiment.

of PAFR stimulation on the apoptosis of osteoclasts was examined using methylcarbamoyl PAF (mc-PAF), a nonhydrolyzable agonist of PAFR. Although a number of osteoclasts promptly became apoptotic after purification, $1 \mu \mathrm{M}$ of mc-PAF enhanced survival (Figure 6A). The administration of $1 \mu \mathrm{M}$ PAF also had a survival effect on the osteoclasts but was less potent than $1 \mu \mathrm{M}$ mc-PAF. This is possibly because PAF is degraded by acetylhydrolases during the 24-hour incubation in serum (18). WEB 2086, an antagonist of PAFR, inhibited the stimulatory effects of mc-PAF on the survival of osteoclasts (Figure 6B). M-CSF is well known to induce the survival of osteoclasts (19). Simultaneous administration of mc-PAF and $\mathrm{M}$-CSF increased the osteoclast survival rate more than a sole administration of mc-PAF or M-CSF (Figure 6B). The stimulatory effects of mc-PAF on the survival of osteoclasts were not found in osteoclasts of PAFR-KO mice (Figure 6C). IL-1 $\beta$ also enhanced the survival of osteoclasts and their Ca resorptive activity, and these parameters were significantly suppressed by WEB 2086 treatment (Figure 7, A and $\mathrm{B}$ ) and genetic PAFR deficiency (Figure 7, C and D).

Next, we examined the effect of PAFR on IL- $1 \beta$-mediated bone resorption in an organ culture system of parietal bones.

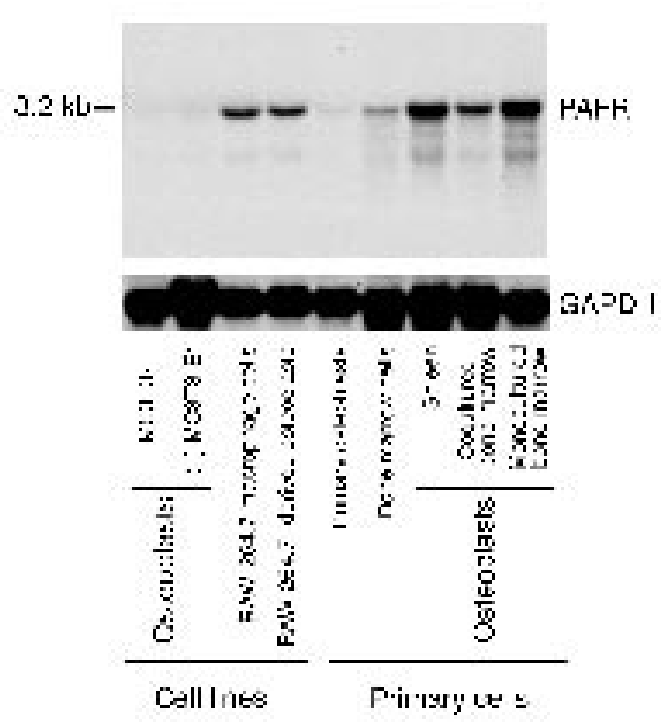



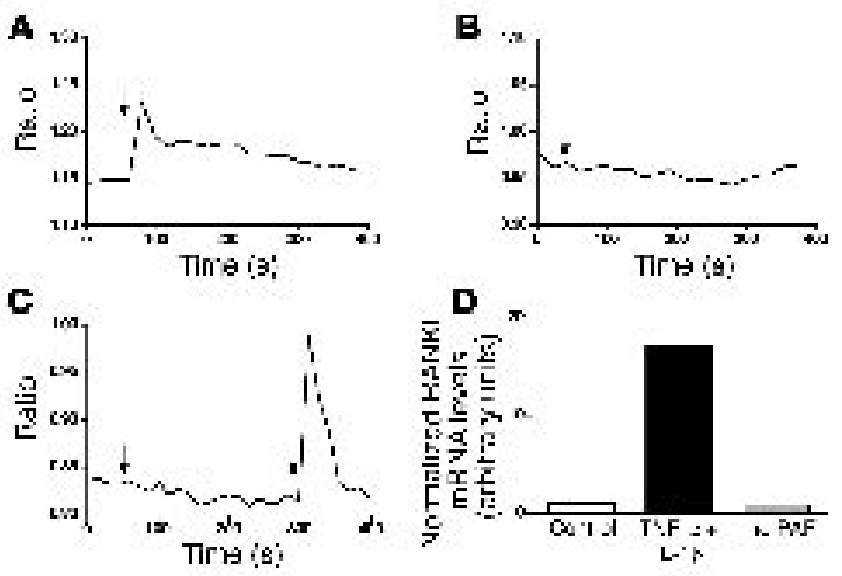

Semiquantitative grading of the cultured parietal bones revealed an increase in the degree of osteoclastic bone resorption after IL-1 $\beta$ treatment. In line with the results of the bone cell experiments described above (Figure 7), both WEB 2086 treatment and genetic ablation of PAFR conferred resistance to osteoclastic resorption in the cultured bones (Figure 8).

\section{Discussion}

This study is the first to demonstrate the critical effects of PAF on bone resorption in a murine model of postmenopausal osteoporosis (Figure 1). Markedly ameliorated bone resorption after ovariectomy in PAFR-KO mice suggests that PAF enhances bone resorption through a receptor. Ovariectomy did not affect indices related to bone volume (BV/TV, Tb.N, and Tb.Sp) or those related to bone turnover (O.Th and Oc.S/BS) in PAFR-KO mice (Figure 2). Prevention of increased O.Th may result from restrained bone turnover. PAFR deficiency did not affect bone turnover in mice with an intact ovarian function, as indicated by observations that shamoperated PAFR-KO mice showed similar bone volume and bone turnover to sham-operated PAFR-WT mice. Therefore, it is unlikely that PAF is required for normal trabecular bone metabolism.

It is interesting to note that PAFR deficiency completely prevented osteoporotic bone resorption. The prevention of bone loss

\section{Figure 5}

No response of osteoblasts to PAF or mc-PAF. (A) PAF (100 nM) elevated the $\left[\mathrm{Ca}^{2+}\right]_{i}$ level in spleen-derived osteoclasts from PAFR-WT mice. (B) PAF did not elevate the $\left[\mathrm{Ca}^{2+}\right]_{i}$ level in spleen-derived osteoclasts from PAFR-KO mice. (C) PAF did not evoke the $\left[\mathrm{Ca}^{2+}\right]_{i}$ response in osteoblasts that were reactive to ATP $(10 \mu \mathrm{M}$, positive control). The arrow denotes the point of PAF administration. The dotted arrow indicates the point of ATP administration. Ratio: $340-$ to $380-\mathrm{nm}$ fluorescence quotient. These results are the representative traces of single cells measured repeatedly. Total numbers of cells measured in two independent experiments were 38, 69 , and 34 for osteoclasts from PAFR-WT mice, osteoclasts from PAFR$\mathrm{KO}$ mice, and osteoblasts from PAFR-WT mice, respectively. Among the monitored cells, 38, 0 , and 0 cells responded to PAF. (D) Treatment with mc-PAF $(1 \mu \mathrm{M})$ had no effect on the mRNA expression of RANKL in osteoblasts, while TNF- $\alpha$ and IL-1 $\beta$ (10 ng/ml each) increased about 20fold. Values shown were measured in duplicate and normalized to $\beta$-actin mRNA expression. The experiment was performed twice independently with similar results.

observed after ovariectomy was reported in mice lacking TNF- $\alpha$ (20), IL-1 receptor (21), osteopontin (22), iNOS (23), and histidine decarboxylase (3). Therefore, these molecules, including PAFR, seem necessary, but not sufficient, for bone resorption following ovariectomy, because a deficiency in a single molecule entirely rescued the phenotype. As PAFR is activated by oxidized phospholipids $(6,24)$, these ligands may also be involved in bone resorption.

PAF is synthesized via two major pathways: the de novo pathway and the remodeling pathway (13). In response to extracellular stimuli, cells produce PAF via the remodeling pathway involving $\mathrm{PLA}_{2}$ and lyso-PAF acetyltransferase. We previously reported that, among at least 19 isozymes, $\mathrm{CPLA}_{2} \alpha$ played a dominant role in the production of PAF in Ca ionophore-stimulated murine peritoneal exudate cells $(14,15)$. In the present investigation, we showed that osteoclasts had more prominent $\mathrm{CPLA}_{2} \alpha$ expression and higher lysoPAF acetyltransferase activity compared with osteoblasts (Figure 3). These data indicate that PAF is produced mainly by osteoclasts in response to extracellular stimuli. However, the PAF production is below the detection limit in osteoclasts (data not shown), possibly because only a small number of osteoclasts were available, and PAF is rapidly degraded by acetylhydrolase enzyme in the culture system.

Estrogen is considered to preserve bone mass through the modulation of bone cell lifespan (25) and to decrease cytokine-
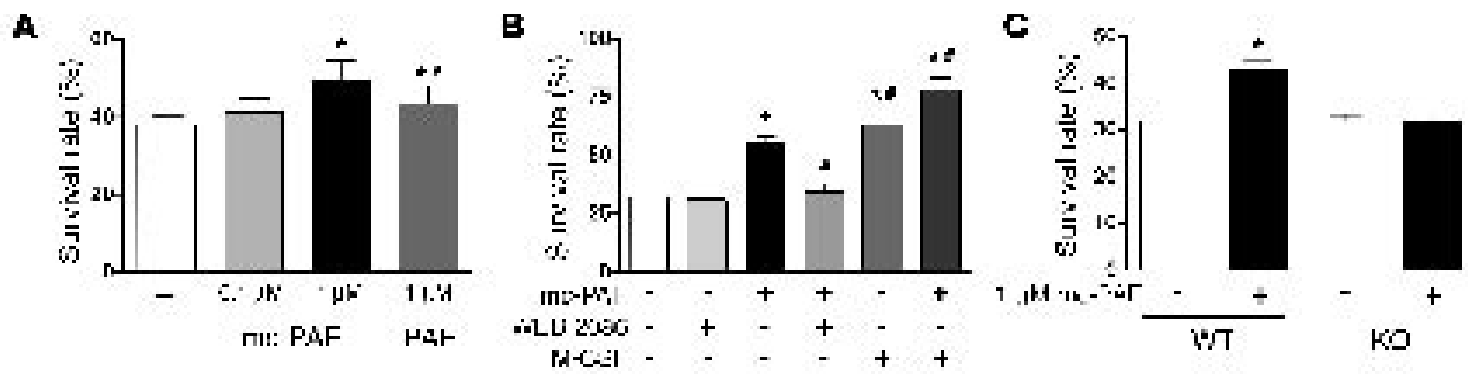

\section{Figure 6}

Survival effect of PAF on osteoclasts. (A) A number of osteoclasts promptly receded after purification. Addition of $1 \mu \mathrm{M}$ mc-PAF, a nonhydrolyzable agonist of PAFR, to the isolated osteoclast cultures significantly stimulated the survival of osteoclasts. The administration of $1 \mu \mathrm{M}$ PAF also had the survival effect, although PAF was less potent than mc-PAF. (B) WEB $2086(10 \mu \mathrm{M})$ inhibited the stimulatory effects of mc-PAF on the survival of osteoclasts. M-CSF $(100 \mathrm{ng} / \mathrm{ml})$ and mc-PAF $(1 \mu \mathrm{M})$ synergistically increased the osteoclast survival. (C) The stimulatory effects of $\mathrm{mc}-\mathrm{PAF}(1 \mu \mathrm{M})$ on the survival of osteoclasts were not found in osteoclasts from PAFR-KO mice. Data are expressed as mean \pm SD of five replicate cultures. The experiment was performed twice independently with similar results. The survival rate was calculated by dividing the number of surviving osteoclasts at 24 hours by the number of osteoclasts at 0 hours. ${ }^{\star} P<0.0001$ vs. control; $\# P<0.01$ vs. mc-PAF-treated osteoclasts. 

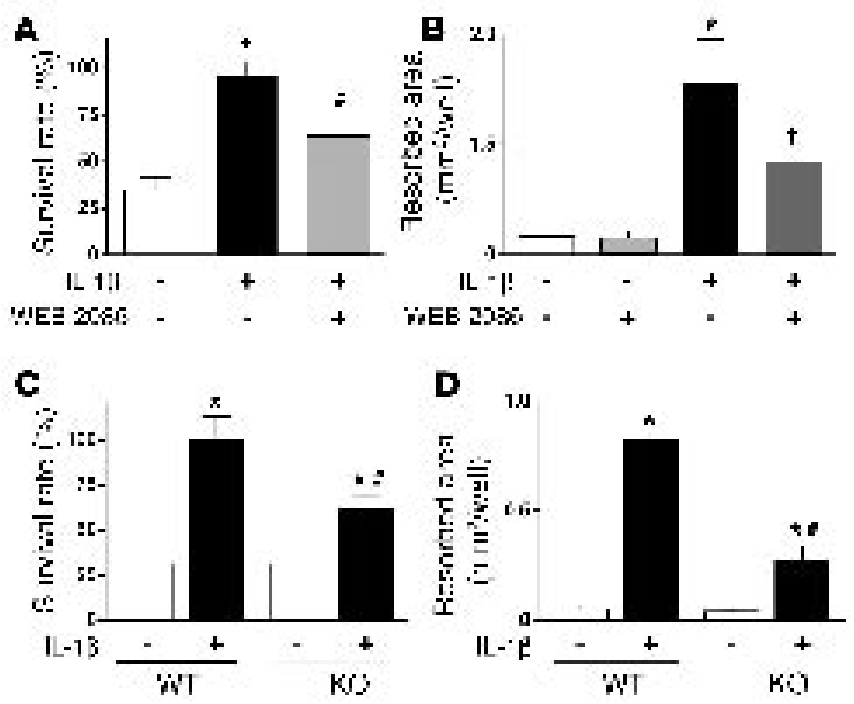

Figure 7

Effect of PAFR on IL-1 $\beta$-induced osteoclast survival and Ca resorption. (A) Effect of WEB 2086 on osteoclast survival stimulated by IL-1 $\beta$. WEB $2086(10 \mu \mathrm{M})$ inhibited the stimulatory effects of IL-1 $\beta(1 \mathrm{ng} / \mathrm{ml})$ on the survival of osteoclasts, while WEB 2086 alone did not have any effects. Similar results were obtained in two independent experiments. ${ }^{*} P<0.0001$ vs. control; ${ }^{\#} P<0.0001$ vs. IL-1 $\beta$-treated osteoclasts. (B) Effect of WEB 2086 on Ca resorption by osteoclasts treated with IL-1 $\beta$. In an osteoblast/osteoclast coculture system, WEB $2086(10 \mu \mathrm{M})$ suppressed the stimulatory effect of IL-1 $\beta(1 \mathrm{ng} / \mathrm{ml})$ on Ca resorption of osteoclasts. WEB 2086 alone did not affect Ca resorption. Similar results were obtained in three independent experiments. ${ }^{*} P<0.0001$ vs. control. ${ }^{\dagger} P<0.005$ vs. IL-1 $\beta$-treated osteoclasts. (C) Survival of PAFR-deficient osteoclasts stimulated by IL-1 $\beta$. When treated with 1 $\mathrm{ng} / \mathrm{ml} \mathrm{IL-1} \beta$, osteoclasts from PAFR-KO mice displayed a significantly lower survival rate than those from PAFR-WT mice. Similar results were obtained in two independent experiments. ${ }^{*} P<0.0001 \mathrm{vs}$. control; ${ }^{\#} P<0.0001$ vs. IL-1 $\beta$-treated PAFR-WT osteoclasts. (D) Ca resorption by PAFR-deficient osteoclasts treated with IL-1 $\beta$. When treated with 0.3 $\mathrm{ng} / \mathrm{ml} \mathrm{IL-1} \beta$, osteoclasts from PAFR-KO mice displayed a significantly lower Ca resorption activity than those from PAFR-WT mice. Similar results were obtained in two independent experiments. ${ }^{*} P<0.0001$ vs. control. ${ }^{\sharp} P<0.0001$ vs. IL-1 $\beta$-treated PAFR-WT osteoclasts.

driven osteoclastogenesis (26). TNF- $\alpha$ and IL-1 are among the most powerful stimulants of bone resorption and are also wellrecognized inhibitors of bone formation (27-29). Studies of women after surgical ovariectomy or natural menopause revealed that estrogen withdrawal is associated with an increased production of these cytokines $(30,31)$. We showed here that TNF- $\alpha$ and IL-1 $\beta$ increased lyso-PAF acetyltransferase activity in osteoclasts. These results suggest that increased levels of TNF- $\alpha$ and IL-1 after ovariectomy result in enhanced PAF production by osteoclasts.

Osteoblasts/stromal cells and osteoclasts are the principal cells in the bone-remodeling system. Numerous systemic hormones, such as parathyroid hormone, 1,25-dihydroxyvitamin D3 [1,25$(\mathrm{OH})_{2} \mathrm{D}_{3}$ ], cytokines, and growth factors modify the bone-resorptive response of osteoclasts through a primary action on the osteoblasts/stromal cells $(19,32)$. In contrast, only a few factors are known to act directly on osteoclasts; calcitonin inhibits and soluble RANKL stimulates osteoclast bone-resorbing activities without the activation of osteoblasts/stromal cells $(33,34)$. While osteoblasts expressed a small amount of PAFR mRNA, osteoclasts expressed high levels (Figure 4). Consistent with the mRNA expression profiles, the level of $\left[\mathrm{Ca}^{2+}\right]_{i}$ was elevated in PAFR-WT osteoclasts in response to PAF; no Ca response was observed in osteoblasts or PAFR-KO osteoclasts (Figure 5, B and C). In addition, mc-PAF had no effect on the mRNA expression of RANKL in osteoblasts (Figure 5D). Thus, PAF may be a novel factor that acts on osteoclasts in an autocrine/paracrine manner without affecting osteoblasts.

We demonstrated that PAF has a survival effect on osteoclasts through PAFR (Figure 6). The survival of mature osteoclasts relies on several factors, such as RANKL, IL-1, TNF- $\alpha$, and M-CSF (35). Furthermore, TNF-binding protein and decoy IL-1 receptor reduced bone loss due to estrogen deficiencies $(36,37)$. Several intracellular signaling pathways, including the activations of extracellular signal regulated kinase $(16,38)$, NF-кB (39), and PI3K $(16,40)$ have been shown to be involved in the survival of osteoclasts. PAF is reported to activate these signaling molecules in several cell lines (7) and to evoke Ca responses in osteoclasts (41). While the intracellular signaling pathways of PAFR that regulate cell survival in osteoclasts remain to be elucidated, PAF is likely to affect the resorptive activity by increasing osteoclast number.

We do not have a clear explanation for the data that $1 \mu \mathrm{M} \mathrm{mc-PAF}$ or PAF was required for the survival assay (Figure 6A). A 24-hour incubation may result in the chemical and enzymic degradation of the compounds. Nevertheless, these effects are receptor-mediated processes as they were not observed in WEB 2086-treated or PAFRKO cells (Figure 6, B and C). It is of interest that both treatment with PAFR antagonist and the genetic ablation of PAFR reduced survival rates of IL-1 $\beta$-stimulated osteoclasts (Figure 7, A and C). These data suggest that IL- $1 \beta$ promotes the production of PAF, which in turn increases osteoclast survival through PAFR.

It is possible that PAF enhances osteoclastogenesis, osteoclast recruitment, and resorptive activity in vivo because positive effects of PAF on osteoclast motility and resorptive activity in vitro have been reported $(12,41)$. Our study indeed demonstrated that WEB 2086 treatment and PAFR deficiency reduced the stimulatory effect of IL-1 $\beta$ on Ca resorption in the coculture system of both osteoclasts and osteoblasts (Figure 7, B and D). Therefore, PAFR activation may lead to enhancement of osteoclastic bone resorption activity and/or osteoclast motility in addition to prolongation of cell survival. As to osteoclastogenesis, we were not able to detect any stimulatory effects of PAF on RAW 264.7 cells or cocultured bone marrow cells (data not shown). Moreover, there is a possibility of an indirect mechanism through which PAFR may alter osteoclastic resorption. For example, PAF may act on other bone marrow cells, for example, the B lymphocyte lineage to induce osteoclastogenesis.

To establish an experimental link between the phenotypes of ovariectomized mice and the in vitro data from isolated bone cells, the organ culture experiments were conducted with parietal bones. Consequently, we found that PAFR mediated the IL- $1 \beta$-stimulated bone resorption (Figure 8), the data further supporting a role for the PAF-PAFR system in the exacerbation of osteoporosis.

In conclusion, we propose a model for PAF action in bone resorption (Figure 9). Withdrawal of estrogen after ovarian dysfunction increases the levels of cytokines such as TNF- $\alpha$ and IL-1 in blood and bone marrow, as described previously $(30,31,42)$. These cytokines appear to enhance PAF production in osteoclasts by activating lyso-PAF acetyltransferase. Thus, PAF activates PAFR on osteoclasts in an autocrine/paracrine manner and affects osteoclastic cell functions including cell survival, cell motility, and 


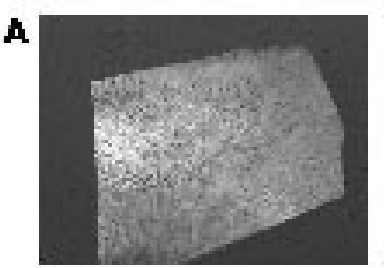

Cnrro

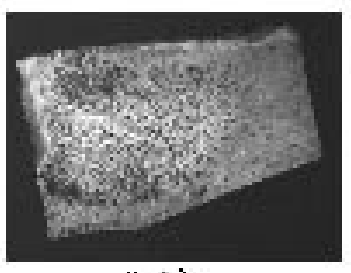

II-1)

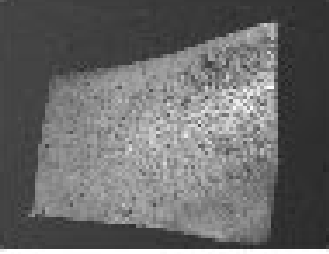

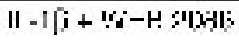

B

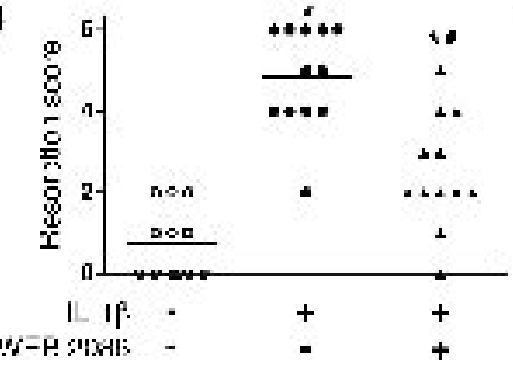

c

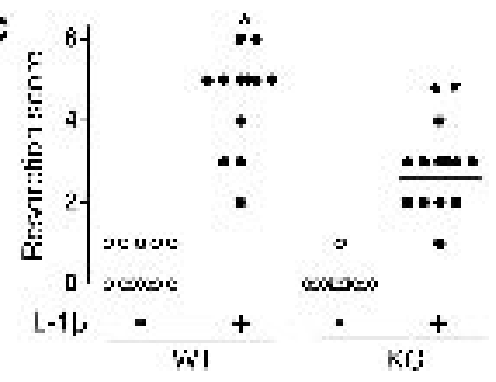

\section{Figure 8}

Effect of PAFR on osteoclastic resorption stimulated by IL-1 $\beta$. (A) Radiographic images of parietal bones cultured in the presence of IL-1 $\beta(0.3$ $\mathrm{ng} / \mathrm{ml})$ and/or WEB $2086(100 \mu \mathrm{M})$. Scale bar: 1.0 $\mathrm{mm}$. (B and $\mathbf{C}$ ) Assessments of osteoclastic bone resorption. The resorption levels of the cultured parietal bones were semiquantitatively scored as described in Methods, and scores for individual bone tissues are presented in each graph. Bars depict means of groups. IL-1 $\beta$ treatment greatly enhanced the bone resorption. Both WEB 2086 (B) and PAFR deficiency (C) significantly suppressed the stimulatory effect of IL-1 $\beta$. ${ }^{*} P<0.0001$ vs. control; $\# P<0.005$ vs. IL-1 $\beta$-treated osteoclasts in (B) or IL-1 $\beta$-treated PAFR-WT osteoclasts in (C). bone-resorption activity. The autocrine/paracrine action of PAF on osteoclasts is noteworthy because PAF could be a candidate for targeting the prevention of osteoporosis without affecting osteoblasts. Furthermore, these findings suggest that PAF inhibition would be beneficial for diseases such as periodontitis, arthritis, and hypercalcemia of malignancy, in which PAF is abundantly produced $(9-11,43)$.

\section{Methods}

Materials. $\alpha \mathrm{MEM}$ was purchased from Invitrogen Corp. (Carlsbad, California, USA) and FBS from Bioserum (Victoria, Australia). A monoclonal antibody directed against mouse $\mathrm{CPLA}_{2} \alpha$ (catalog no. sc-454) was obtained from Santa Cruz Biotechnology Inc. (Santa Cruz, California, USA), and peroxidase-conjugated anti-mouse IgG antibody from Amersham Biosciences Corp. (Piscataway, New Jersey, USA). Recombinant murine M-CSF and murine soluble RANKL were from Techne Inc. (Burlington, New Jersey, USA) and PeproTech (London, United Kingdom), respectively. Recombinant human TNF- $\alpha$ and human IL- $1 \beta$ were from Dainippon Pharmaceutical Co. Ltd. (Osaka, Japan) and Techne Inc., respectively. Bacterial collagenase from Clostridium histolyticum and $1,25-(\mathrm{OH})_{2} \mathrm{D}_{3}$ were purchased from Wako Pure Chemicals Industries Inc. (Osaka, Japan). Dispase and collagen gel (cell matrix type IA) were from Nitta Gelatin Inc. (Osaka, Japan). $\left[{ }^{3} \mathrm{H}\right]$ Acetyl-coenzyme A (acetyl-CoA), $185 \mathrm{GBq} / \mathrm{mmol}$, was from Amersham Biosciences. PAF (C16) and 1-O-hexadecyl-sn-glycero-3-phosphocholine (lyso-PAF, C16) were from Cayman Chemical (Ann Arbor, Michigan, USA). WEB 2086 was a generous gift from Boehringer Ingelheim $\mathrm{GmbH}$ (Ingelheim, Germany). Bondapak C-8 (dimethyloctylsilyl-bonded amorphous silica, 37-55 $\mu \mathrm{m}$ ) was from Millipore Corp. (Bedford, Massachusetts, USA). Naphtol AS-MX phosphate was purchased from Sigma-Aldrich (St. Louis, Missouri, USA), and dissolved in $\mathrm{N}, \mathrm{N}$-dimethylformamide from Sigma-Aldrich. Other chemicals were of analytical grade.

Mice. All animal studies were conducted in accordance with the guidelines for animal research at The University of Tokyo and were approved by the University of Tokyo Ethics Committee for Animal Experiments. PAFR-KO mice were established using a gene-targeting strategy (44). PAFR-KO mice and the corresponding PAFR-WT mice have been backcrossed for ten generations onto a C57BL/6N genetic background. Mice were fed with a standard laboratory diet and water ad libitum. Mice aged 8-11 weeks old were used. PAFRKO mice had body weights (mean $\pm \mathrm{SD}=18.8 \pm 2.4 \mathrm{~g}, n=9$ ) indistinguishable from those of PAFR-WT mice (17.2 $\pm 2.0 \mathrm{~g}, n=11)$. C57BL/6N mice from Charles River Japan (Tokyo, Japan) were also used for in vitro studies.

Ovariectomy. Age-matched PAFR-KO and PAFR-WT female mice received a bilateral ovariectomy or a sham procedure in which the ovaries were exteriorized but not removed. Mice were sacrificed 4 weeks after the surgical procedure.

Radiographic analysis. Radiographs of the femurs and tibiae were taken by soft x-ray (model CMB-2; SOFTEX Co. Ltd., Tokyo, Japan). The BMD of the femurs was measured by DXA (model DCS-600R; ALOKA Co. Ltd., Tokyo, Japan).The BMD was calculated by dividing the bone mineral content of the measured area by the area.

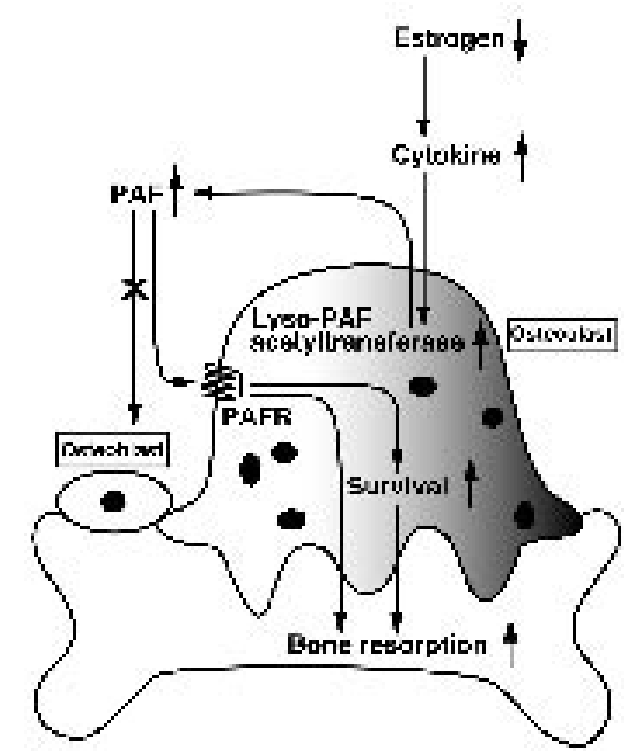

Figure 9

Proposed model of PAF action in bone resorption. For details, see Discussion. 
Histomorphometry. Tibiae were fixed with $70 \%$ ethanol and embedded in glycol methacrylate, and undecalcified $3-\mu \mathrm{m}$ sections were prepared. The sections were stained with toluidine blue and subjected to histomorphometric analyses using a light microscope with a micrometer and an image analyzer (Osteoplan II; Carl Zeiss Japan, Tokyo, Japan). BV/TV (\%), Tb.N (per mm), Tb.Sp ( $\mu \mathrm{m})$, O.Th $(\mu \mathrm{m})$, and Oc.S/BS (\%) were measured in an area $1.2 \mathrm{~mm}$ in length from $0.3 \mathrm{~mm}$ below the growth plate at the proximal metaphysis of the tibiae. The quantitative histomorphometric analyses were performed in a blinded fashion.

Osteoblast culture. Calvariae dissected from 1-day-old mice were washed in PBS and digested with $0.1 \%$ collagenase and $0.2 \%$ dispase for $10 \mathrm{~min}$ five times, and cells from fractions 2-5 were pooled and cultured for 7 days in $\alpha$ MEM containing 10\% FBS. MC3T3-E1 mouse osteogenic cells (RIKEN, Saitama, Japan) were also cultured in $\alpha$ MEM containing $10 \%$ FBS. On the sixth day of culture, mcPAF $(1 \mu \mathrm{M})$, TNF- $\alpha(10 \mathrm{ng} / \mathrm{ml})$, and IL- $1 \beta(10 \mathrm{ng} / \mathrm{ml})$ were added upon the medium exchange. Osteoblasts both with and without cytokine stimulation were cultured for another day.

Osteoclast culture. Spleens were collected from 7- to 8-week-old male mice and forced through a $40-\mu \mathrm{m}$ nylon mesh cell strainer (Falcon, Franklin Lakes, New Jersey, USA). Bone marrow was flushed from the femurs and tibiae of 7- to 8-week-old male mice. Osteoclasts are differentiated from hematopoietic cell lineages in bone marrow cultures by stimulating with RANKL and M-CSF $(45,46)$. Spleen cells or bone marrow cells were cultured in $\alpha$ MEM containing $10 \%$ FBS with soluble RANKL $(100 \mathrm{ng} / \mathrm{ml})$ and M-CSF $(10 \mathrm{ng} / \mathrm{ml})$ for 7 days. To differentiate into osteoclasts, RAW 264.7 mouse macrophage cells were cultured in DMEM containing $10 \%$ FBS with soluble RANKL (100 ng/ml) for 5 days (47, 48). Osteoclasts were treated with TNF- $\alpha$ and IL-1 $\beta$ as described for osteoblasts. For the detection of living osteoclasts, cells were stained with trypan blue and subsequently reacted with $0.01 \%$

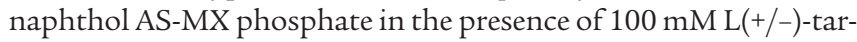
taric acid ( $\mathrm{pH}$ 5.0) for detecting tartrate-resistant acid phosphatase (TRAP) activity. Trypan blue-negative and TRAP-positive cells with more than three nuclei were counted as living osteoclasts. Of the total number of cells, 95\%, 99\%, and 99\% were osteoclasts, and $99 \%, 100 \%$, and $100 \%$ were TRAP positive in spleen, bone marrow, and RAW 264.7 cell-derived cell culture systems, respectively.

Measurement of lyso-PAF acetyltransferase activity. The collected cells, in a buffer containing $20 \mathrm{mM}$ Tris- $\mathrm{HCl}$ ( $\mathrm{pH} 7.4), 0.3 \mathrm{M}$ sucrose, 5 $\mathrm{mM}$ 2-mercaptoethanol, $20 \mu \mathrm{M}$ 4-amidinophenylmethanesulfonyl fluoride (APMSF; Sigma-Aldrich) and $1 \times$ Complete (a protease inhibitor mixture; Roche Diagnostics GmbH, Mannheim, Germany), were sonicated and centrifuged at $800 \mathrm{~g}$ for $10 \mathrm{~min}$. The supernatant was used for the enzyme reaction, which was performed in a 96-well round-bottom microplate (no. 25850; Corning Inc., Corning, New York, USA) in a water bath at $37^{\circ} \mathrm{C}$. The reaction mixture contained $20 \mathrm{mM}$ Tris-HCl (pH 7.4), 5 mM 2-mercaptoethanol, $3 \mathrm{mM} \mathrm{CaCl}_{2}, 20$ $\mu \mathrm{M}$ APMSF, $1 \mathrm{mg} / \mathrm{ml}$ phosphatidylcholine (Sigma-Aldrich), and 100 $\mu \mathrm{M}\left[{ }^{3} \mathrm{H}\right]$ acetyl-CoA $(1.11 \mathrm{GBq} / \mathrm{mmol})$ with or without $20 \mu \mathrm{M}$ lyso$\mathrm{PAF}$ in a total volume of $0.1 \mathrm{ml}$. After a $15-\mathrm{min}$ incubation, $0.1 \mathrm{ml}$ of methanol was added to terminate the reaction. The radioactivity of Bondapack C-8 resin ( $2 \mathrm{mg}$ )-adsorptive products were measured according to the method of Kume et al. (49), except for washing the resin one time before and eight times after application of the reaction mixture with 50\% methanol in $20 \mathrm{mM}$ Tris- $\mathrm{HCl}$ ( $\mathrm{pH}$ 7.4). The enzyme activity was estimated by the increase in the radioactivity due to the addition of lyso-PAF to the reaction mixture.
Western blot analysis. Cells were lysed with a buffer containing 50 mM Tris- $\mathrm{HCl}$ (pH 7.4), $10 \mathrm{mM} \mathrm{MgCl}_{2}, 0.25 \mathrm{M}$ sucrose, and $1 \times$ Complete. The protein concentration in the cell lysate was measured using a Protein Assay Kit (Bio-Rad Laboratories, Hercules, California, USA) using BSA (fraction V, fatty acid-free; Sigma-Aldrich) as a standard. Sixteen micrograms of protein was separated by $8 \%$ polyacrylamide gel electrophoresis and transferred to a nitrocellulose membrane. After blocking with Block Ace (Dainippon Pharmaceutical Co. Ltd.), the membrane was incubated with a mouse monoclonal antibody against $\mathrm{CPLA}_{2} \alpha$ and then with peroxidase-conjugated anti-mouse IgG antibody. Immunoreactive proteins were visualized by the ECL chemiluminescence reaction (Amersham Biosciences) following the manufacturer's instructions.

Northern blot analysis. Cells were lysed with Isogen (Wako Pure Chemicals Industries Inc.) for the total RNA extraction. Ten micrograms of total RNA was separated by $0.8 \%$ agarose $/ 0.66 \mathrm{M}$ formaldehyde gel electrophoresis, and transferred to a Hybond-N+ membrane as described previously (44). The membrane was hybridized with either the PAFR cDNA probe or the human GAPDH cDNA probe as described (44). The final washing condition was $1 \times$ SSC and $0.1 \% \mathrm{SDS}$ for $30 \mathrm{~min}$ at $65^{\circ} \mathrm{C}$. The membrane was exposed to an $x$-ray film (Fuji Photo Film Co., Tokyo, Japan) with an intensifying screen for 24 hours at $-80^{\circ} \mathrm{C}$.

Detection of RANKL $m R N A$ by real-time quantitative RT-PCR. Total cDNAs were synthesized from $2 \mu \mathrm{g}$ of total RNA of the cultured primary osteoblasts by oligo(dT) priming using Superscript II reverse transcriptase (Invitrogen Corp.). Primers for RANKL were: 5'-CCTGAGGCCCAGCCATTT- $3^{\prime}$ and $5^{\prime}$-CTTGGCCCAGCCTCGAT-3' (50). Primers for $\beta$-actin were: $5^{\prime}$-GCTGTGCTATGTTGCTCTAGACTT- $3^{\prime}$ and ' 5'-AATTGAATGTAGTTTCATGGATGC-3'. A capillary real-time thermocycler (LightCycler; Roche Diagnostics $\mathrm{GmbH}$ ) was used to perform quantitative PCR. In a $20-\mu$ f final volume, $0.02 \mu \mathrm{l}$ of the cDNA solution was mixed with LightCycler-FastStart DNA Master SYBR Green I (Roche Diagnostics $\mathrm{GmbH}$ ), $3 \mathrm{mM} \mathrm{MgCl}$, and $0.5 \mu \mathrm{M}$ each of sense and antisense primers. The mixture was used as a template for the amplification by the following protocol: a denaturing step at $95^{\circ} \mathrm{C}$ for 3 $\mathrm{min}$, then an amplification and quantification program of RANKL repeated for 50 cycles of $95^{\circ} \mathrm{C}$ for $15 \mathrm{~s}, 56^{\circ} \mathrm{C}$ for $5 \mathrm{~s}$, and $72^{\circ} \mathrm{C}$ for $4 \mathrm{~s}$, or that of $\beta$-actin repeated for 45 cycles of $95^{\circ} \mathrm{C}$ for $15 \mathrm{~s}, 58^{\circ} \mathrm{C}$ for $5 \mathrm{~s}$, and $72^{\circ} \mathrm{C}$ for $8 \mathrm{~s}$. SYBR Green I fluorescence was measured, and relative quantification was made against $\beta$-actin cDNA used as an internal standard.

Measurement of $\left[\mathrm{Ca}^{2+}\right]_{\mathrm{i}}$. The $\left[\mathrm{Ca}^{2+}\right]_{i}$ levels within individual cells were analyzed as described previously (51). Briefly, after washing with PBS, cells were loaded with $4 \mu \mathrm{M}$ fura-2/acetoxymethyl ester (Dojindo Laboratories, Kumamoto, Japan) in PBS containing 0.5 $\mathrm{mM} \mathrm{MgCl} 2$ and $1 \mathrm{mM} \mathrm{CaCl}_{2}$ at $37^{\circ} \mathrm{C}$ for $30 \mathrm{~min}$, rinsed twice with the buffer, and then incubated at $37^{\circ} \mathrm{C}$ for an additional $10 \mathrm{~min}$. The recorded fluorescence images were digitized using an online image processor (Olympus Corp., Tokyo, Japan). The change in the 340- to 380-nm fluorescence ratio was recorded.

Osteoclast survival assay. A coculture system of mouse osteoblasts and bone marrow cells has been developed to examine the regulatory mechanisms of osteoclast function $(46,52)$. Removal of osteoblasts/stromal cells from cocultures induces apoptosis of osteoclasts $(19,52)$. Mouse osteoblasts $\left(1 \times 10^{6}\right.$ cells/dish $)$ and bone marrow cells $\left(2 \times 10^{7}\right.$ cells/dish $)$ from femurs and tibiae of 7 - to 8 -week-old C57BL $/ 6 \mathrm{~N}$ mice were prepared as described above and were cocultured on $10-\mathrm{cm}$ culture dishes coated with $0.24 \%$ 
collagen gel matrix. The culture medium, $\alpha$ MEM with $10 \%$ FBS, $100 \mathrm{nM} 1,25-(\mathrm{OH})_{2} \mathrm{D}_{3}$, and $1 \mu \mathrm{M} \mathrm{PGE}_{2}$, was changed every 2-3 days. After 7 days of culture, dishes were treated with $4 \mathrm{ml}$ of $0.2 \%$ collagenase in $\alpha \mathrm{MEM}$ for $20 \mathrm{~min}$ at $37^{\circ} \mathrm{C}$, and the collected cells were suspended in $\alpha \mathrm{MEM}$ containing $10 \%$ FBS. An aliquot of the crude osteoclast preparation was cultured in 24-well plates. After incubation for 2 hours, the plates were treated with PBS containing $0.1 \%$ collagenase and $0.2 \%$ dispase to remove osteoblasts/stromal cells. After the purification procedure, $88 \%$ of the total cells were osteoclasts and $97 \%$ were TRAP positive. The purified osteoclasts were cultured with mc-PAF $(1 \mu \mathrm{M}), \mathrm{M}-\mathrm{CSF}(100 \mathrm{ng} / \mathrm{ml})$, or IL-1 $\beta$ $(1 \mathrm{ng} / \mathrm{ml})$ for 24 hours in the presence or absence of WEB 2086 $(10 \mu \mathrm{M})$. To calculate the survival rate, the numbers of surviving osteoclasts were counted under light microscopy (BH-2; Olympus, Corp.) before and after the incubation.

Ca resorption assay. An aliquot of cocultured osteoblast/osteoclast preparation with $\alpha$ MEM containing $10 \%$ FBS $(0.25 \mathrm{ml})$ was transferred into a $\mathrm{CaPO}_{4}$-coated dish (BioCoat Osteologic bone culture system; BD Biosciences, Bedford, Massachusetts, USA). After 48hour culture with $0.3-1.0 \mathrm{ng} / \mathrm{ml} \mathrm{IL-1} \beta$ and/or $10 \mu \mathrm{M}$ WEB 2086, cells were removed with a bleach solution $(6 \% \mathrm{NaOCl}$ and $5.2 \%$ $\mathrm{NaCl})$. The dishes were washed with water and photographed under light microscopy (BH-2; Olympus). The resorbed area $\left(\mathrm{mm}^{2}\right)$ with cleared $\mathrm{CaPO}_{4}$ resorption pits was measured using Photoshop 7.0 software (Adobe Systems Inc., San Jose, California, USA).

Organ culture of parietal bone. The bone organ culture was performed as described previously (53). Briefly, paired parietal bones from 1-day-old mice were aseptically harvested and cultured for 24 hours in $2 \mathrm{ml} \mathrm{BGJ}$ medium (Invitrogen Corp.) containing 1 $\mathrm{mg} / \mathrm{ml}$ BSA (fraction V; Serologicals Proteins Inc., Kankakee, Illinois, USA). Then, each half parietal bone was transferred to fresh medium containing $0.3 \mathrm{ng} / \mathrm{ml}$ IL-1 $\beta$ and/or $100 \mu \mathrm{M}$ WEB 2086, and cultured for an additional 2 days with a medium change on the following day. To detect bone resorption, parietal bones were fixed with $70 \%$ ethanol and processed for soft x-ray analysis. A semiquantitative scoring system was used to grade the bone resorption, where +3 signified large and widespread resorption in the majority of bone surface, and +1 signified a small number of tiny resorption pits. Randomized and blinded photographs were graded by T. Shimizu and S. Ishii, and the resorption score represents the sum of the two scores.

Statistical analysis. Means of groups were compared by analysis of variance, and significance of differences was determined by post hoc testing using Fisher's PLSD method (StatView; SAS Institute Inc., Cary, North Carolina, USA). Two group comparisons for the resorption scores were performed by using Mann-Whitney's $U$-test. A $P$ value less than 0.05 was taken to have statistical significance.

\section{Acknowledgments}

We would like to thank T. Satoh, C. Ohkawara, and Y. Yamada for their technical assistance; Y. Kita for his instruction in ovariectomy; and T. Yokomizo, N. Uozumi, T. Okuno, A. Toda, S.M. Tokuoka, Y. Ide, Y. Kihara, and K. Noguchi (Department of Biochemistry and Molecular Biology) for their support and valuable suggestions. We are also grateful to N. Koshikiya (Department of Oral and Maxillofacial Surgery) for his help in $\left[\mathrm{Ca}^{2+}\right]_{i}$ measurements. This work was supported in part by Grants-in-Aid from the Ministry of Education, Science, Culture, Sports and Technology of Japan (to T. Shimizu, T. Takato, S. Ishii, and H. Hikiji). This work was also supported by Grants-in-Aid for Comprehensive Research on Aging and Health and for Research on Allergic Disease and Immunology from the Ministry of Health, Labor and Welfare of Japan and grants from the Yamanouchi Foundation for Research on Metabolic Disorders, the Kanae Foundation for Life \& Socio-medical Science, the Uehara Memorial Foundation (to S. Ishii), and Daiwa Securities Health Foundation (to H. Hikiji).

Received for publication November 10, 2003, and accepted in revised form April 27, 2004.

Address correspondence to: Satoshi Ishii, Department of Biochemistry and Molecular Biology, Faculty of Medicine, The University of Tokyo, Hongo 7-3-1, Bunkyo, Tokyo 113-0033, Japan. Phone: 81-35802-2925; Fax: 81-3-3813-8732; E-mail: mame@m.u-tokyo.ac.jp.
1. Turner, R.T., Riggs, B.L., and Spelsberg, T.C. 1994. Skeletal effects of estrogen. Endocr. Rev. 15:275-300.

2. Sherman, S. 2001. Preventing and treating osteoporosis: strategies at the millennium. Ann. N. Y. Acad. Sci. 949:188-197.

3. Fitzpatrick, L.A., et al. 2003. Targeted deletion of histidine decarboxylase gene in mice increases bone formation and protects against ovariectomyinduced bone loss. Proc. Natl. Acad. Sci. U. S. A. 100:6027-6032.

4. Yoshida, K., et al. 2002. Stimulation of bone formation and prevention of bone loss by prostaglandin $\mathrm{E}$ EP4 receptor activation. Proc. Natl. Acad. Sci. U. S. A. 99:4580-4585.

5. Hanahan, D.J. 1986. Platelet activating factor: a biologically active phosphoglyceride. Annu. Rev. Biochem. 55:483-509.

6. Prescott, S.M., Zimmerman, G.A., Stafforini, D.M., and McIntyre, T.M. 2000. Platelet-activating factor and related lipid mediators. Annu. Rev. Biochem. 69:419-445.

7. Ishii, S., and Shimizu, T. 2000. Platelet-activating factor (PAF) receptor and genetically engineered PAF receptor mutant mice. Prog. Lipid. Res. 39:41-82.

8. Honda, Z., Ishii, S., and Shimizu, T. 2002. Plateletactivating factor receptor. J. Biochem. 131:773-779.

9. Noguchi, K., Morita, I., and Murota, S. 1989. The detection of platelet-activating factor in inflamed human gingival tissue. Arch. Oral. Biol. 34:37-41.

10. Pettipher, E.R., Higgs, G.A., and Henderson, B 1987. PAF-acether in chronic arthritis. Agents. Actions. 21:98-103.

11. Nigam, S., Muller, S., and Benedetto, C. 1989. Elevated plasma levels of platelet-activating factor (PAF) in breast cancer patients with hypercalcemia. J. Lipid. Mediat. 1:323-328.

12. Zheng, Z.G., Wood, D.A., Sims, S.M., and Dixon, S.J. 1993. Platelet-activating factor stimulates resorption by rabbit osteoclasts in vitro. Am. J. Physiol. 264:E74-E81.

13. Snyder, F. 1995. Platelet-activating factor: the biosynthetic and catabolic enzymes. Biochem. J. 305:689-705.

14. Shindou, H., Ishii, S., Uozumi, N., and Shimizu, T. 2000. Roles of cytosolic phospholipase $\mathrm{A}_{2}$ and platelet-activating factor receptor in the $\mathrm{Ca}$ induced biosynthesis of PAF. Biochem. Biophys. Res. Commun. 271:812-817.

15. Uozumi, N., et al. 1997. Role of cytosolic phospholipase $\mathrm{A}_{2}$ in allergic response and parturition. Nature. 390:618-622.

16. Lee, S.E., et al. 2001. Tumor necrosis factor- $\alpha$ supports the survival of osteoclasts through the activation of Akt and ERK. J. Biol. Chem. 276:49343-49349.

17. Fuller, K., et al. 1993. Macrophage colony-stimulating factor stimulates survival and chemotactic behavior in isolated osteoclasts. J. Exp. Med. 178:1733-1744.

18. Tjoelker, L.W., et al. 1995. Anti-inflammatory properties of a platelet-activating factor acetylhydrolase. Nature. 374:549-553.

19. Udagawa, N., et al. 1999. Osteoblasts/stromal cells stimulate osteoclast activation through expression of osteoclast differentiation factor/RANKL but not macrophage colony-stimulating factor: receptor activator of NF-кB ligand. Bone. 25:517-523.

20. Roggia, C., et al. 2001. Up-regulation of TNF-producing $\mathrm{T}$ cells in the bone marrow: a key mechanism by which estrogen deficiency induces bone loss in vivo. Proc. Natl. Acad. Sci. U. S. A. 98:13960-13965.

21. Lorenzo, J.A., et al. 1998. Mice lacking the type I interleukin-1 receptor do not lose bone mass after ovariectomy. Endocrinology. 139:3022-3025.

22. Yoshitake, H., Rittling, S.R., Denhardt, D.T., and Noda, M. 1999. Osteopontin-deficient mice are resistant to ovariectomy-induced bone resorption. Proc. Natl. Acad. Sci. U. S. A. 96:8156-8160.

23. Cuzzocrea, S., et al. 2003. Inducible nitric oxide synthase mediates bone loss in ovariectomized mice. Endocrinology. 144:1098-1107.

24. Smiley, P.L., Stremler, K.E., Prescott, S.M., Zimmerman, G.A., and McIntyre, T.M. 1991. Oxidatively fragmented phosphatidylcholines activate human neutrophils through the receptor for platelet-activating factor. J. Biol. Chem. 266:11104-11110. 
25. Kameda, T., et al. 1997. Estrogen inhibits bone resorption by directly inducing apoptosis of the bone-resorbing osteoclasts. J. Exp. Med. 186:489-495.

26. Cenci, S., et al. 2000. Estrogen deficiency induces bone loss by enhancing T-cell production of TNF- $\alpha$. J. Clin. Invest. 106:1229-1237.

27. Stashenko, P., Dewhirst, F.E., Rooney, M.L., Desjardins, L.A., and Heeley, J.D. 1987. Interleukin-1 $\beta$ is a potent inhibitor of bone formation in vitro. J. Bone Miner. Res. 2:559-565.

28. Bertolini, D.R., Nedwin, G.E., Bringman, T.S., Smith, D.D., and Mundy, G.R. 1986. Stimulation of bone resorption and inhibition of bone formation in vitro by human tumour necrosis factors. Nature. 319:516-518.

29. Thomson, B.M., Mundy, G.R., and Chambers, T.J. 1987. Tumor necrosis factors $\alpha$ and $\beta$ induce osteoblastic cells to stimulate osteoclastic bone resorption. J. Immunol. 138:775-779.

30. Pacifici, R., et al. 1991. Effect of surgical menopause and estrogen replacement on cytokine release from human blood mononuclear cells. Proc. Natl. Acad. Sci. U. S. A. 88:5134-5138.

31. Pacifici, R., et al. 1987. Spontaneous release of interleukin 1 from human blood monocytes reflects bone formation in idiopathic osteoporosis. Proc. Natl. Acad. Sci. U. S. A. 84:4616-4620.

32. Suda, T., Ueno, Y., Fujii, K., and Shinki, T. 2003. Vitamin D and bone. J. Cell. Biochem. 88:259-266.

33. Yasuda, H., et al. 1998. Osteoclast differentiation factor is a ligand for osteoprotegerin/osteoclastogenesis-inhibitory factor and is identical to TRANCE/RANKL. Proc. Natl. Acad. Sci. U. S. A. 95:3597-3602.

34. Body, J.J. 2002. Calcitonin for the long-term prevention and treatment of postmenopausal osteoporosis. Bone. 30:75S-79S.

35. Suda, K., Woo, J.T., Takami, M., Sexton, P.M., and
Nagai, K. 2002. Lipopolysaccharide supports survival and fusion of preosteoclasts independent of TNF- $\alpha$, IL-1, and RANKL. J. Cell. Physiol. 190:101-108.

36. Ammann, P., et al. 1997. Transgenic mice expressing soluble tumor necrosis factor-receptor are protected against bone loss caused by estrogen deficiency. J. Clin. Invest. 99:1699-1703.

37. Sunyer, T., Lewis, J., Collin-Osdoby, P., and Osdoby, P. 1999. Estrogen's bone-protective effects may involve differential IL-1 receptor regulation in human osteoclast-like cells. J. Clin. Invest. 103:1409-1418.

38. Miyazaki, T., et al. 2000. Reciprocal role of ERK and NF-KB pathways in survival and activation of osteoclasts. J. Cell Biol. 148:333-342.

39. Jimi, E., et al. 1998. Activation of NF- $\mathrm{B}$ is involved in the survival of osteoclasts promoted by interleukin-1. J. Biol. Chem. 273:8799-8805.

40. Wong, B.R., et al. 1999. TRANCE, a TNF family member, activates Akt/PKB through a signaling complex involving TRAF6 and c-Src. Mol. Cell. 4:1041-1049.

41. Wood, D.A., Hapak, L.K., Sims, S.M., and Dixon, S.J. 1991. Direct effects of platelet-activating factor on isolated rat osteoclasts. Rapid elevation of intracellular free calcium and transient retraction of pseudopods. J. Biol. Chem. 266:15369-15376.

42. Kimble, R.B., et al. 1995. Simultaneous block of interleukin-1 and tumor necrosis factor is required to completely prevent bone loss in the early postovariectomy period. Endocrinology. 136:3054-3061.

43. Wiebe, S.H., Hafezi, M., Sandhu, H.S., Sims, S.M., and Dixon, S.J. 1996. Osteoclast activation in inflammatory periodontal diseases. Oral. Dis. 2:167-180.

44. Ishii, S., et al. 1998. Impaired anaphylactic responses with intact sensitivity to endotoxin in mice lacking a platelet-activating factor receptor. J. Exp. Med.
187:1779-1788

45. Sells Galvin, R.J., Gatlin, C.L., Horn, J.W., and Fuson, T.R. 1999. TGF- $\beta$ enhances osteoclast differentiation in hematopoietic cell cultures stimulated with RANKL and M-CSF. Biochem. Biophys. Res. Commun. 265:233-239.

46. Okada, Y., et al. 2000. Prostaglandin G/H synthase2 is required for maximal formation of osteoclastlike cells in culture. J. Clin. Invest. 105:823-832.

47. Matsumoto, Y., et al. 2002. Possible involvement of the vascular endothelial growth factor-Flt-1-focal adhesion kinase pathway in chemotaxis and the cell proliferation of osteoclast precursor cells in arthritic joints. J. Immunol. 168:5824-5831.

48. Battaglino, R., et al. 2002. c-myc is required for osteoclast differentiation. J. Bone Miner. Res. 17:763-773.

49. Kume, K., Waga, I., and Shimizu, T. 1997. Microplate chromatography assay for acetyl-CoA:lysoplatelet-activating factor acetyltransferase. Anal. Biochem. 246:118-122.

50. Cao, J., Venton, L., Sakata, T., and Halloran, B.P. 2003. Expression of RANKL and OPG correlates with age-related bone loss in male C57BL/ 6 mice. J. Bone Miner. Res. 18:270-277.

51. Koizumi, T., et al. 2003. Cell density and growthdependent down-regulation of both intracellular calcium responses to agonist stimuli and expression of smooth-surfaced endoplasmic reticulum in MC3T3-E1 osteoblast-like cells. J. Biol. Chem. 278:6433-6439.

52. Li, X., et al. 2002. p38 MAPK-mediated signals are required for inducing osteoclast differentiation but not for osteoclast function. Endocrinology. 143:3105-3113.

53. Yano, H., Ohya, K., and Amagasa, T. 1994. Effects of insulin on in vitro bone formation in fetal rat parietal bone. Endocr. J. 41:293-300. 